\title{
Permanent Discontinuation of Glucocorticoids in Polymyalgia Rheumatica Is Uncommon but May Be Enhanced by Amino Bisphosphonates
}

\author{
Alessandro Giollo (D), Maurizio Rossini (iD, Francesco Bettili, Francesco Ghellere, \\ Elena Fracassi, Luca Idolazzi, Davide Gatti, and Ombretta Viapiana
}

\begin{abstract}
Objective. The duration of treatment with glucocorticoids (GC) in polymyalgia rheumatica (PMR) is often longterm. Amino bisphosphonates (N-BP) are used in PMR for the prevention of GC-induced osteoporosis, but they coulsd also have immunomodulatory properties. Whether they can be effective as an adjuvant treatment in PMR is unknown. We aimed to establish whether the use of N-BP in our PMR cohort may be associated with GC discontinuation.

Methods. We conducted a retrospective review of all patients diagnosed with PMR recorded in our electronic medical notes. Cox regression analyses were used to examine the association between the use of N-BP and discontinuation of GC.

Results. Data were retrieved for 385 patients (mean age $71 \pm 10$ yrs, 64\% females, mean initial prednisone dose $19 \pm 9 \mathrm{mg}$ /day). The median followup time was 38 months (range 9-57); more than $60 \%$ of patients were exposed to N-BP. GC were discontinued in $47 \%$ of patients after a median time of 20 months (range 14-27), but subsequently restarted in 39\%. Overall, 276/385 patients (72\%) were actively treated at their last available evaluation (mean prednisone dose $4.9 \pm 5.5 \mathrm{mg} /$ day), while $123 / 205(60 \%)$ were still receiving GC after 24 months of followup. The use of N-BP was associated with the discontinuation of GC (adjusted HR 0.66, 95\% CI 0.50-0.88), independent of age, initial GC dose, and osteoporosis.

Conclusion. Unlike current guidelines, longterm treatment with GC is often necessary. These preliminary data suggest that N-BP may be involved in the management of PMR. (First Release November 1 2018; J Rheumatol 2019;46:318-22; doi:10.3899/jrheum.180324)
\end{abstract}

Key Indexing Terms:

POLYMYALGIA RHEUMATICA

GLUCOCORTICOIDS

\section{BISPHOSPHONATE OSTEOPOROSIS}

Polymyalgia rheumatica (PMR) is a chronic inflammatory disease in older adults that causes pain, stiffness, and inflammation of the shoulder and pelvic girdles ${ }^{1}$. It is typically associated with an increase of acute-phase reactants, and up to one-fifth of patients with PMR also have concomitant giant cell arteritis ${ }^{1,2}$. Glucocorticoids (GC) are still currently the mainstay treatment for PMR, and generally it is proposed that

From the Rheumatology Unit, Department of Medicine, University of Verona, Verona, Italy.

A. Giollo, MD, Rheumatology Unit, Department of Medicine, University of Verona; M. Rossini, MD, PhD, Rheumatology Unit, Department of Medicine, University of Verona; F. Bettili, MD, Rheumatology Unit, Department of Medicine, University of Verona; F. Ghellere, MD, Rheumatology Unit, Department of Medicine, University of Verona; E. Fracassi, MD, PhD, Rheumatology Unit, Department of Medicine, University of Verona; L. Idolazzi, MD, PhD, Rheumatology Unit, Department of Medicine, University of Verona; D. Gatti, MD, PhD, Rheumatology Unit, Department of Medicine, University of Verona; O. Viapiana, MD, PhD, Rheumatology Unit, Department of Medicine, University of Verona.

Address correspondence to Dr. A. Giollo, Rheumatology Unit, Department of Medicine, University of Verona, Piazzale L.A. Scuro 10, 37134 Verona, Italy.E-mail: alessandrogiollo@gmail.com

Accepted for publication August 8, 2018.
GC would be discontinued about 1 year after the diagnosis ${ }^{3}$. Despite current guidelines, many patients with PMR are unable to stop GC treatment within this time frame. Observational data showed that $40 \%$ of patients with PMR would continue GC for longer than 4 years ${ }^{4}$. The median duration of GC therapy had indeed been increasing between 1989 and 2008 within the United Kingdom ${ }^{5}$, reaching 57 weeks in men and 64 weeks in women. In the United States, the median time to permanent discontinuation of GC was almost 6 years between 2000 and $2015^{6}$.

These data suggest that there is a reluctance to discontinue GC in patients with $\mathrm{PMR}^{7}$ and our clinical perception agrees with this. PMR is generally considered a benign disease of the elderly; however, GC-related adverse events are a matter of concern. There is an unmet need for identifying the characteristics of those patients with PMR at risk of longterm GC use. GC-related adverse events can occur in up to $85 \%$ of treated cases $^{8,9}$, particularly GC-induced osteoporosis (GIOP) and fractures ${ }^{5}$. A retrospective cohort study conducted using primary care records from the UK-based Clinical Practice Research Datalink showed that the risk of

Personal non-commercial use only. The Journal of Rheumatology Copyright ( $)$ 2019. All rights reserved. 
fracture is increased by $63 \%$ in PMR compared with the control population ${ }^{10}$. For the prevention or treatment of GIOP, amino bisphosphonates (N-BP) are recommended as the first-line option for individuals at moderate or high risk of fracture, alongside calcium and vitamin D supplements ${ }^{11}$.

In more recent years, there has been increasing interest in the analgesic, antiinflammatory, and immunomodulatory effects of $\mathrm{N}-\mathrm{BP}^{12,13}$. In our study, we aimed to establish the proportion of patients with PMR receiving active treatment with GC in our cohort, and the potential predictors of longterm GC use. In particular, we investigated whether the use of N-BP may be associated with GC discontinuation in PMR.

\section{MATERIALS AND METHODS}

Study population and design. Clinical data were collected retrospectively from electronic medical records (EMR) of our rheumatology unit. Data extraction from more than 10,000 EMR was performed in January 2017, returning 467 records containing the entry "polymyalgia rheumatica." The local Ethical Committee approved the study (approval number: CE 1876). Written informed consent from patients to publish the material was not requested for this observational study.

Data collection. Patients were eligible for inclusion if (1) they had at least 2 distinct assessments recorded in their EMR; (2) they had no previous treatment with GC for PMR; and (3) they fulfilled the 2012 European League Against Rheumatism/American College of Rheumatology Classification Criteria for Polymyalgia Rheumatica ${ }^{14}$. The following exclusion criteria were applied: (1) any history of large-vessel vasculitis; and (2) other diagnosis that could explain the symptoms (e.g., rheumatoid arthritis, connective tissue disease, infection, or cancer). Each EMR was reviewed separately to identify the dosage of GC used (in prednisone equivalent), conventional synthetic disease-modifying antirheumatic drugs (csDMARD), concomitant treatment comorbidities, and inflammatory markers [erythrocyte sedimentation rate (ESR) and C-reactive protein (CRP)]. The prescribed GC dosage was tracked from the start of GC until the last available assessment.

Definitions. The followup time was defined as the time between the first dose of GC and the last evaluation available in the records. The time to first GC discontinuation was defined as the time between the first dose and the first discontinuation of GC therapy. Peripheral involvement was defined as a synovitis detected by physical examination or imaging. Disease relapse was defined as an increase in GC dosage owing to symptoms attributed to PMR, inflammatory markers, or both; short bursts of GC used for any other inflammatory conditions were not included. Patients treated with N-BP received 1 or more of alendronate, risedronate, ibandronate, or zoledronate. When prescribed, N-BP are started routinely per standard practice when the first dose of GC is given. Exposure to N-BP was considered only if the drug was continued for at least 3 months for alendronate, risedronate or ibandronate, or if a patient had at least 1 infusion of zoledronate. Osteoporosis was defined as a T score $<2.5$ at spine or hip. Active GC treatment was defined as a patient receiving $\mathrm{GC}$ at the last evaluation available in their EMR.

Statistical analysis. Data are reported as mean values (SD) for continuous variables or percentages for categorical variables. Time intervals are reported as median and interquartile range (IQR). Between-group comparisons of categorical variables were performed by Pearson chi-square test. Univariable and multivariable Cox regression models were used to examine the association between potential predictors and active treatment as the main outcome. Linear relationships between GC dosage and variables of interests were analyzed by Pearson correlation coefficient. Logistic regression was used to identify the characteristics associated with the use of N-BP medications. All statistical analyses were performed using SPSS Version 20 (SPSS Inc.) and statistical significance was identified by 2 -tailed $\mathrm{p}<0.05$.

\section{RESULTS}

Baseline characteristics of patients. The study population consisted of 385 patients (mean age 71 yrs, females 64\%). Baseline characteristics are summarized in Table 1. About $30 \%$ of patients had some peripheral involvement, mostly oligoarticular. Inflammatory markers were increased at baseline with a mean ESR of $49 \mathrm{~mm} / \mathrm{h}$ and CRP of $33 \mathrm{mg} / \mathrm{l}$. The average dosage of prednisone was $19 \mathrm{mg}$ daily (range 2-50 $\mathrm{mg}$ daily), and it was weakly correlated with age $(\mathrm{r}=0.117, \mathrm{p}=0.022)$, hemoglobin $(\mathrm{r}=-0.134, \mathrm{p}=0.009)$, CRP $(r=0.153, p=0.003)$, and ESR $(r=0.121, p=0.017)$. One in every 5 patients was receiving treatment with at least 1 csDMARD during followup, mostly with low-dose weekly methotrexate (MTX; range 7.5-20 mg). More than $60 \%$ of patients were treated with N-BP, of whom $26 \%$ were diagnosed with osteoporosis at baseline.

Characteristics of followup. Data were collected over a median follow period of 37.7 months (IQR 37.9). Among the 385 patients evaluated at baseline, $205(53 \%)$ were followed up over 24 months. Overall, 276 patients $(72 \%)$ were still receiving active treatment with $\mathrm{GC}$ [mean prednisone dose at last visit 4.9 (5.5) $\mathrm{mg}$ daily] at their last available evaluation. Disease flares occurred in 307 patients (80\%), on average 1 (1) flare per patient. GC were discontinued in 180 patients [47\%; mean time to discontinuation 24.0 mos (IQR 20.3)]. Nonetheless, GC were restarted in 71/180 patients (39\%).

Factors associated with persistent treatment with GC. Cox regression analyses were used to establish the factors associated with longterm use of GC, and the results are shown in Table 2. The univariable analysis showed that these factors were associated with persistence in therapy: older age, number of relapses, peripheral involvement, and higher CRP.

Table 1. Baseline clinical characteristics and demographics of 385 patients with polymyalgia rheumatica.

\begin{tabular}{lc}
\hline Variable & Values \\
\hline Age, yrs & $71(10)$ \\
Female sex, $\mathrm{n}(\%)$ & $246(64)$ \\
Peripheral joint involvement, n (\%) & $113(29)$ \\
Hemoglobin, g/dl & $13(1)$ \\
ESR, mm/h & $49(25)$ \\
CRP, mg/l & $33(34)$ \\
Initial prednisone dosage, mg daily & $19(9)$ \\
csDMARD at baseline, n (\%) & $86(22)$ \\
Methotrexate, n (\%) & $69(18)$ \\
Hydroxychloroquine, n $(\%)$ & $19(5)$ \\
N-BP, n (\%) & $240(62)$ \\
\end{tabular}

Values are mean (SD) unless otherwise specified. CRP: C-reactive protein; csDMARD: conventional synthetic disease-modifying antirheumatic drugs; ESR: erythrocyte sedimentation rate; SD: standard deviation; N-BP: amino bisphosphonates.

Personal non-commercial use only. The Journal of Rheumatology Copyright @ 2019 . All rights reserved. 
Table 2. Univariable and multivariable analyses of factors associated with a persistent treatment with glucocorticoids (Cox regression).

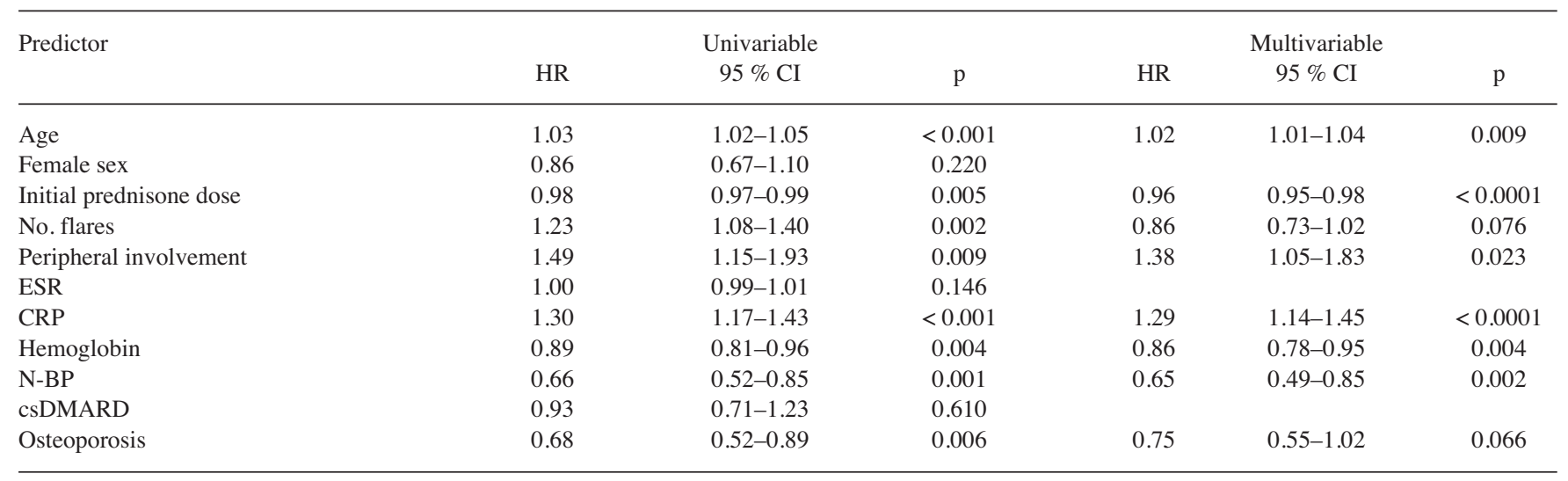

ESR: erythrocyte sedimentation rate; CRP: C-reactive protein; csDMARD: conventional synthetic disease-modifying antirheumatic drugs; N-BP: amino bisphosphonates.

These were associated with discontinuation: a higher initial dosage of GC, higher hemoglobin, osteoporosis, and the use of N-BP. Except for osteoporosis, all predictors significantly associated with the outcome at univariable analysis were confirmed in multivariable analysis (Table 2). The use of N-BP had the higher association with the outcome (Figure
1). Moreover, the positive association between N-BP and GC discontinuation retained statistical significance even after the exclusion of patients with peripheral involvement or treatment with MTX (data not shown). A subanalysis only of patients with a followup over 24 months confirmed the significant association between N-BP and treatment duration

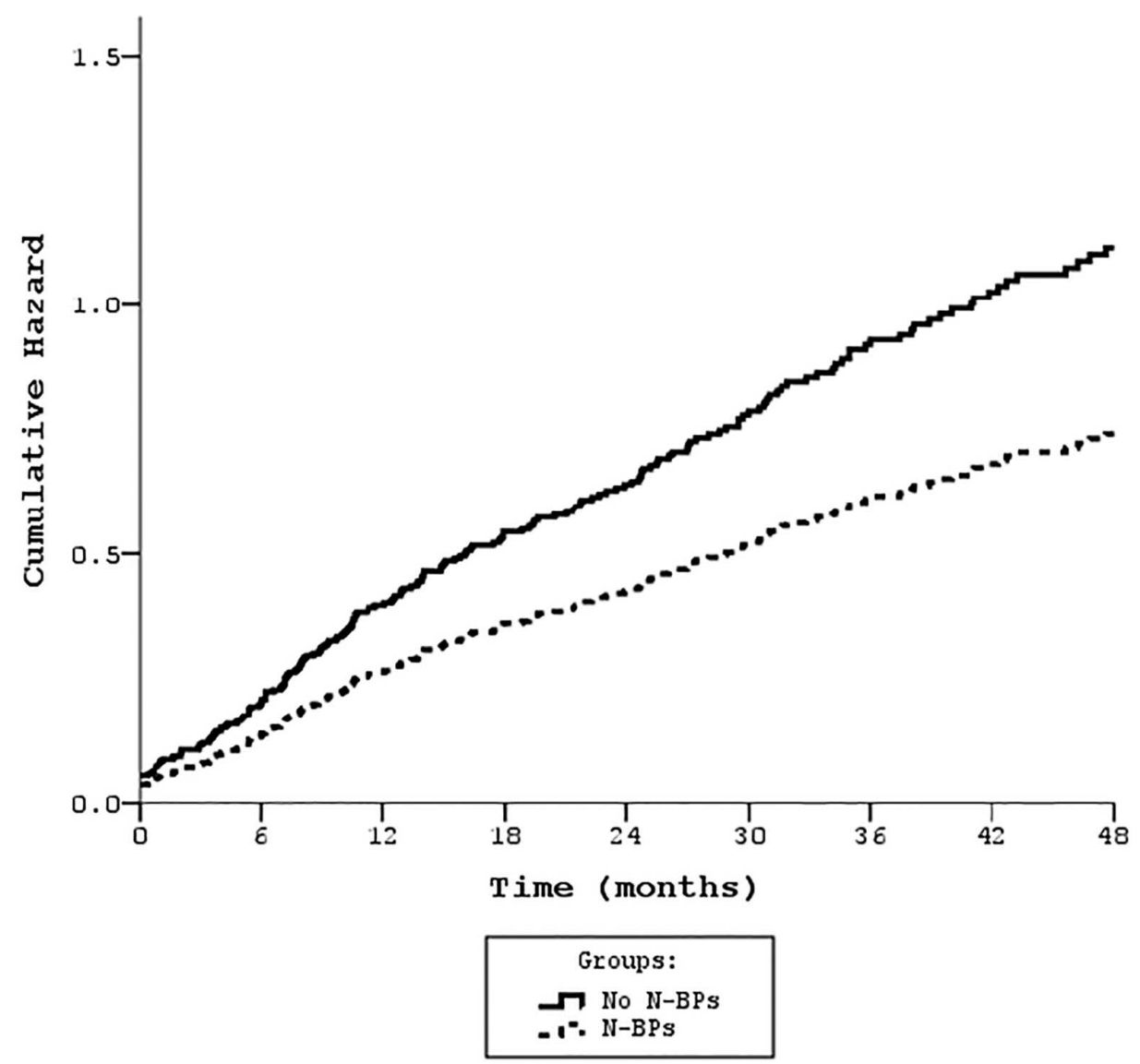

Figure 1. Risk function of longterm glucocorticoid use for the use of amino bisphosphonates (N-BP); adjusted HR 0.65 (95\% CI 0.49-0.85), $\mathrm{p}=0.002$. 
(adjusted HR 0.61, 95\% CI 0.38-0.96). Multiple logistic regression analysis showed that the patients treated with $\mathrm{N}-\mathrm{BP}$ were more frequently older (OR $1.05,95 \% \mathrm{CI}$ $1.02-1.08, \mathrm{p}<0.001)$ and female (OR 2.85, 95\% CI $1.63-4.80, \mathrm{p}<0.001)$. Hemoglobin levels (OR 1.24, 95\% CI $1.00-1.54, \mathrm{p}=0.047)$ and CRP (OR 1.34, 95\% CI 1.04-1.74, $\mathrm{p}=0.026$ ) were correlated with the use of N-BP, irrespective of the diagnosis of osteoporosis at baseline.

\section{DISCUSSION}

The first remarkable finding of our study is that more than $70 \%$ of patients with PMR were still receiving GC at their last clinical evaluation. Although our study design also included patients with a short followup, hence more likely to still be receiving treatment, it should be stated that $60 \%$ of patients followed over 2 years were receiving active treatment with GC.

Therefore, most patients with PMR are at risk of becoming chronic GC users, and they will encounter serious adverse events that can occur in the long term even with a low dose (i.e., $<7.5 \mathrm{mg}$ daily) of prednisone. In line with previous experiences ${ }^{15,16,17}$, these patients had clinical features of a more severe disease course (higher inflammatory markers, lower hemoglobin, peripheral joint involvement, and frequent relapses), suggesting that this subgroup of patients with PMR may benefit the most from alternative treatments to GC.

Novel approaches to spare GC in PMR have been investigated, albeit with unconvincing or very preliminary results ${ }^{3,18}$. Considering this, an innovative approach of our study is investigating whether the use of N-BP can affect the clinical outcome of PMR. N-BP is prescribed in 13-80\% 10,19 of GC-treated cases. Only $62.3 \%$ of our study patients had been prescribed N-BP, despite GIOP being a well-known risk factor for frailty fractures and that the Italian National Health System reimburses it as a prophylactic treatment. Nevertheless, we found that our patients with PMR treated with N-BP had on average a $35 \%$ lower risk of persisting on active treatment with GC during their followup.

Several studies have investigated the relationships among inflammatory diseases, pathways targeted by N-BP, and the immune system. The use of N-BP has been associated with longterm alterations in peripheral white blood cells and particularly of some subtypes, $\gamma \delta \mathrm{T}$ cells, which have been associated with the acute-phase response (APR) ${ }^{20,21,22}$. The use of N-BP is occasionally associated with APR and it is linked to the activation of $\gamma \delta$ T cells and the release of the pyrogenic cytokines such as interferon- $\gamma$ and tumor necrosis factor- $\alpha$. We observed that the proportion of circulating $\gamma \delta$ $T$ cells is an important determinant of the occurrence of APR after administration of N-BP, and that N-BP treatment is associated with a decrease in circulating $\gamma \delta$ T cells. Further, in patients with osteoporosis treated with bisphosphonates for 1 year, a significant reduction of Th17 pathways [interleukin (IL)-6, IL-17, and IL-23] and elevation of Treg cytokine cascade (IL-10, transforming growth factor- $\beta$ ) can occur $^{23}$. Interestingly, it has been shown that in PMR there may be an imbalance in the Th17:Treg ratio skewed toward Th17, possibly contributing to its pathogenesis ${ }^{24}$.

Of note, the use of N-BP was associated with a shorter duration of treatment independent of the initial dose of prednisone, which was only marginally associated with the outcome. Not surprisingly, the use of N-BP was associated with osteoporosis, which was in turn associated with the hazard of GC discontinuation, because a treatment with N-BP is more likely in these patients. Female sex was not associated with longterm GC use, in contrast with previous studies ${ }^{25,26}$, but consistent with the more frequent use of $\mathrm{N}-\mathrm{BP}$ in women. Accordingly, the missed correlation between female sex and active GC treatment that has been observed in previous research ${ }^{27,28}$ might be explained by the greater proportion of women who could have benefitted from N-BP agents.

We acknowledge that our study, though original, has several limitations. First, the retrospective design does not allow for the assumption of a causal relationship between the use of N-BP and the outcome. Second, the method we used for extracting data from our cohort has not been validated. Finally, some population characteristics such as the initial prednisone dose could have led to biased results, and other individual factors (e.g., concomitant osteoporosis) could have driven the clinician's decision to discontinue GC treatment. Nonetheless, our observational study has some strengths, including the number of patients (one of the highest among observational studies in PMR), and investigation of N-BP as potential disease modifiers in PMR, which has never been analyzed before, to our knowledge.

Longterm treatment with GC in PMR is often necessary despite current guidelines recommending GC discontinuation after a relatively short-term course. However, this finding has no immediate justification and the results of our study encourage investigation of other treatments than GC for the management of PMR. Considering this, N-BP may have be involved in the management of PMR, and further research on this topic is warranted. It would be of interest to analyze both the incidence of PMR in patients exposed to N-BP and the clinical effects of their use in patients with PMR.

\section{REFERENCES}

1. Buttgereit F, Dejaco C, Matteson EL, Dasgupta B. Polymyalgia rheumatica and giant cell arteritis: a systematic review. JAMA 2016;315:2442-58.

2. González-Gay MA, Matteson EL, Castaneda S. Polymyalgia rheumatica. Lancet 2017;390:1700-12.

3. Dejaco C, Brouwer E, Mason JC, Buttgereit F, Matteson EL, Dasgupta B. Giant cell arteritis and polymyalgia rheumatica: current challenges and opportunities. Nat Rev Rheumatol 2017;13:578-92.

4. Ayoub WT, Franklin CM, Torretti D. Polymyalgia rheumatica. Duration of therapy and long-term outcome. Am J Med 1985;79:309-15.

5. Fardet L, Petersen I, Nazareth I. Prevalence of long-term oral

Personal non-commercial use only. The Journal of Rheumatology Copyright @ 2019 . All rights reserved. 
glucocorticoid prescriptions in the UK over the past 20 years. Rheumatology 2011;50:1982-90.

6. Shbeeb I, Challah D, Raheel S, Crowson CS, Matteson EL. Comparable rates of glucocorticoid-associated adverse events in patients with polymyalgia rheumatica and comorbidities in the general population. Arthritis Care Res 2018;70:643-7.

7. Yates M, Watts RA, Swords F, MacGregor AJ. Glucocorticoid withdrawal in polymyalgia rheumatica: the theory versus the practice. Clin Exp Rheumatol 2017;35:1-2.

8. Gabriel SE, Sunku J, Salvarani C, O'Fallon WM, Hunder GG. Adverse outcomes of antiinflammatory therapy among patients with polymyalgia rheumatica. Arthritis Rheum 1997;40:1873-8.

9. Mazzantini M, Torre C, Miccoli M, Baggiani A, Talarico R, Bombardieri S, et al. Adverse events during longterm low-dose glucocorticoid treatment of polymyalgia rheumatica: A retrospective study. J Rheumatol 2012;39:552-7.

10. Paskins Z, Whittle R, Sultan AA, Muller S, Blagojevic-Bucknall M, Helliwell $\mathrm{T}$, et al. Risk of fracture among patients with polymyalgia rheumatica and giant cell arteritis: a population-based study. BMC Med 2018;16:4.

11. Buckley L, Guyatt G, Fink HA, Cannon M, Grossman J, Hansen KE, et al. 2017 American College of Rheumatology guideline for the prevention and treatment of glucocorticoid-induced osteoporosis. Arthritis Care Res 2017;69:1095-110.

12. Le Goff B, Berthelot JM, Maugars Y, Romas E. Alternative use of bisphosphonate therapy for rheumatic disease. Curr Pharm Des 2010;16:3045-52.

13. Viapiana O, Gatti D, Idolazzi L, Fracassi E, Adami S, Troplini S, et al. Bisphosphonates vs infliximab in ankylosing spondylitis treatment. Rheumatology 2014;53:90-4.

14. Dasgupta B, Cimmino MA, Maradit-Kremers H, Schmidt WA, Schirmer M, Salvarani C, et al. 2012 provisional classification criteria for polymyalgia rheumatica: a European League Against Rheumatism/American College of Rheumatology collaborative initiative. Ann Rheum Dis 2012;71:484-92.

15. Salvarani C, Cantini F, Niccoli L, Macchioni P, Consonni D, Bajocchi G, et al. Acute-phase reactants and the risk of relapse/recurrence in polymyalgia rheumatica: A prospective followup study. Arthritis Rheum 2005;53:33-8.

16. Lee JH, Choi ST, Kim JS, Yoon BY, Kwok SK, Kim HS, et al. Clinical characteristics and prognostic factors for relapse in patients with polymyalgia rheumatica (PMR). Rheumatol Int 2013; $33: 1475-80$.
17. Matteson EL, Dejaco C. Polymyalgia rheumatica. Ann Intern Med 2017;166:ITC65-ITC80.

18. Dejaco C, Singh YP, Perel P, Hutchings A, Camellino D, Mackie S, et al; European League Against Rheumatism; American College of Rheumatology. 2015 recommendations for the management of polymyalgia rheumatica: a European League Against Rheumatism/American College of Rheumatology collaborative initiative. Ann Rheum Dis 2015;74:1799-807.

19. Rossini M, Viapiana O, Vitiello M, Malavolta N, La Montagna G, Maddali Bongi S, et al. Prevalence and incidence of osteoporotic fractures in patients on long-term glucocorticoid treatment for rheumatic diseases: the Glucocorticoid Induced OsTeoporosis TOol (GIOTTO) study. Reumatismo 2017;69:30-9.

20. Rossini M, Adami S, Viapiana O, Tripi G, Zanotti R, Ortolani R, et al. Acute phase response after zoledronic acid is associated with long-term effects on white blood cells. Calcif Tissue Int 2013;93:249-52.

21. Rossini M, Adami S, Viapiana O, Ortolani R, Vella A, Fracassi E, et al. Circulating $\gamma \delta \mathrm{T}$ cells and the risk of acute-phase response after zoledronic acid administration. J Bone Miner Res 2012;27:227-30.

22. Rossini M, Adami S, Viapiana O, Fracassi E, Ortolani R, Vella A, et al. Long-term effects of amino-bisphosphonates on circulating $\gamma \delta \mathrm{T}$ cells. Calcif Tissue Int 2012;91:395-9.

23. Talaat RM, Sidek A, Mosalem A, Kholief A. Effect of bisphosphonates treatment on cytokine imbalance between TH17 and Treg in osteoporosis. Inflammopharmacology 2015;23:119-25.

24. Samson M, Audia S, Fraszczak J, Trad M, Ornetti P, Lakomy D, et al. Th1 and th17 lymphocytes expressing cd161 are implicated in giant cell arteritis and polymyalgia rheumatica pathogenesis. Arthritis Rheum 2012;64:3788-98.

25. Cimmino MA, Parodi M, Caporali R, Montecucco C. Is the course of steroid-treated polymyalgia rheumatica more severe in women? Ann N Y Acad Sci 2006;1069:315-21.

26. Mackie SL, Hensor EM, Haugeberg G, Bhakta B, Pease CT. Can the prognosis of polymyalgia rheumatica be predicted at disease onset? Results from a 5-year prospective study. Rheumatology 2010;49:716-22.

27. Muratore F, Pazzola G, Pipitone N, Salvarani C. Recent advances in the diagnosis and treatment of polymyalgia rheumatica. Expert Rev Clin Immunol 2016;12:1037-45.

28. Kermani TA, Warrington KJ. Polymyalgia rheumatica. Lancet 2013;381:63-72. 